\title{
Symmetric matrices, Catalan paths, and correlations
}

\author{
Bernd Sturmfels ${ }^{1}$, Emmanuel Tsukerman $^{1}$, and Lauren Williams ${ }^{1}$ \\ ${ }^{1}$ Department of Mathematics, University of California, Berkeley, CA 94720-3840, USA
}

\begin{abstract}
Kenyon and Pemantle (2014) gave a formula for the entries of a square matrix in terms of connected principal and almost-principal minors. Each entry is an explicit Laurent polynomial whose terms are the weights of domino tilings of a half Aztec diamond. They conjectured an analogue of this parametrization for symmetric matrices, where the Laurent monomials are indexed by Catalan paths. In this paper we prove the Kenyon-Pemantle conjecture, and apply this to a statistics problem pioneered by Joe (2006). Correlation matrices are represented by an explicit bijection from the cube to the elliptope.

Résumé. Kenyon et Pemantle (2014) ont donné une formule pour les entrées d'une matrice carrée en termes des mineurs connectés principaux et presque principaux. Chaque entrée est un polynôme de Laurent explicite dont les termes sont les poids de pavages domino d'un demi-diamant Aztec. Ils ont conjecturé un analogue de cette paramétrisation pour les matrices symétriques, où les monômes de Laurent sont indexés par des chemins catalans. Dans cet article, nous prouvons la conjecture de Kenyon-Pemantle, et se rapportent à un problème de statistiques mis au point par Joe (2006). Les matrices de corrélation sont représentés par une bijection explicite du cube à l'elliptope.
\end{abstract}

Keywords. Symmetric matrix, minors, Catalan path, correlation matrix

\section{Introduction}

In this paper we present a formula for each entry of a symmetric $n \times n$ matrix $X=\left(x_{i j}\right)$ as a Laurent polynomial in $\left(\begin{array}{c}n+1 \\ 2\end{array}\right)$ distinguished minors of $X$. Our result verifies a conjecture of Kenyon and Pemantle from [3]. Let $I$ and $J$ be subsets of $[n]=\{1,2, \ldots, n\}$ with $|I|=|J|$. Let $X_{I}^{J}$ denote the minor of $X$ with row indices $I$ and column indices $J$. Here the indices in $I$ and $J$ are always taken in increasing order. We will employ shorthand notation $i J:=\{i\} \cup J$. The following signed minors will be used:

$$
\begin{aligned}
p_{I} & :=(-1)^{\lfloor|I| / 2\rfloor} \cdot X_{I}^{I} \\
\text { and } \quad a_{i j \mid I} & :=(-1)^{\lceil|I| / 2\rceil} \cdot X_{i I}^{j I} \quad \text { for } i, j \notin I, \quad i \neq j .
\end{aligned}
$$

We call $p_{I}$ and $a_{i j \mid I}$ the principal and almost-principal minors, respectively. The minors $p_{I}, a_{i j \mid I}$ and $a_{j i \mid I}$ are called connected if $1 \leq i<j \leq n$ and $I=\{i+1, i+2, \ldots, j-2, j-1\}$. The $1 \times 1$-minors $a_{i j}:=a_{i j \mid \emptyset}=x_{i j}$ and $p_{k}=x_{k k}$ are connected when $|i-j|=1$ and $1 \leq k \leq n$.

A Catalan path $C$ is a path in the $x y$-plane which starts at $(0,0)$ and ends on the $x$-axis, always stays at or above the $x$-axis, and consists of steps northeast $(1,1)$ and southeast $(1,-1)$. We say that $C$ has size 
$n$ if its endpoints have distance $2 n-2$ from each other. Let $\mathscr{C}_{n}$ denote the set of Catalan paths of size $n$. Its cardinality equals the Catalan number

$$
\left|\mathscr{C}_{n}\right|=\frac{1}{n}\left(\begin{array}{c}
2 n-2 \\
n-1
\end{array}\right), \quad \text { which is } 1,2,5,14,42,132,429,1430,4862 \text { for } n=2, \ldots, 10
$$

Let $G_{n}$ denote the planar graph whose vertices are the $\left(\begin{array}{c}n+1 \\ 2\end{array}\right)$ lattice points $(x, y)$ with $x \geq y \geq 0$ and $x+y \leq 2 n-2$ even, and edges are northeast and southeast steps. Thus $\mathscr{C}_{n}$ consists of the paths from $(0,0)$ to $(2 n-2,0)$ in $G_{n}$. We label the nodes and regions of $G_{n}$ as follows. We assign label $j$ to the node $(2 j-2,0)$, label $a_{i j \mid I}$ to the node $(i+j-2, j-i)$, and label $p_{I}$ to the region below that node. The set $I$ is the numbers between $i$ and $j$. Thus, connected principal and almost-principal minors of $X$ are identified in the graph $G_{n}$ with regions and nodes strictly above the $x$-axis.

The weight $W_{\mathscr{C}}(C)$ of a Catalan path $C$ is a Laurent monomial, derived from the drawing of $C$ in the graph $G_{n}$. Its numerator is the product of the labels $a_{i j \mid I}$ of the nodes of $G_{n}$ that are local maxima or local minima of $C$, and its denominator is the product of the labels $p_{I}$ of the regions which are either immediately below a local maximum or immediately above a local minimum. Thus $W_{\mathscr{C}}(C)$ is a Laurent monomial of degree $\leq 1$. There is no lower bound on the degree due to minima on the $x$-axis; for instance, $\frac{a_{13 \mid 2} a_{35 \mid 4} a_{57 \mid 6} a_{79 \mid 8}}{p_{2} p_{3} p_{4} p_{5} p_{6} p_{7} p_{8}}$ has degree -3 and appears for $n=9$.

The following result was conjectured by Kenyon and Pemantle in [3, Conjecture 1].

Theorem 1.1 The entries of an $n \times n$ symmetric matrix $X=\left(x_{i j}\right)$ satisfy the identity

$$
x_{i j}=\sum_{C} W_{\mathscr{C}}(C)
$$

where the sum is over all Catalan paths $C$ between node $i$ and node $j$ in $G_{n}$

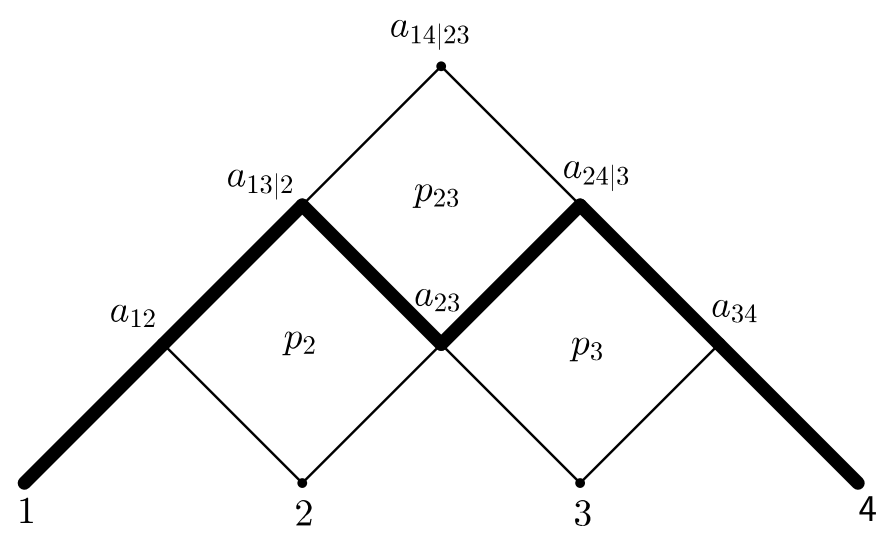

Fig. 1: A Catalan path $C$ in the planar graph $G_{4}$ with weight $\frac{a_{13 \mid 2} a_{23} a_{24 \mid 3}}{p_{2} p_{23} p_{3}}$. 
For symmetric matrices of size $n=4$, Theorem 1.1 states the following formula:

$$
X=\left(\begin{array}{cccc}
p_{1} & a_{12} & \frac{a_{13 \mid 2}}{p_{2}}+\frac{a_{12} a_{23}}{p_{2}} & \frac{a_{14 \mid 23}}{p_{23}}+\frac{a_{12} a_{24 \mid 3}}{p_{2} p_{3}}+\frac{a_{13 \mid 2} a_{34}}{p_{2} p_{3}}+\frac{a_{12} a_{23} a_{34}}{p_{2} p_{3}}+\frac{a_{13 \mid 2} a_{23} a_{24 \mid 3}}{p_{2} p_{23} p_{3}} \\
* & p_{2} & a_{23} & \frac{a_{24 \mid 3}}{p_{3}}+\frac{a_{23} a_{34}}{p_{3}} \\
* & * & p_{3} & a_{34} \\
* & * & * & p_{4}
\end{array}\right)
$$

The entry $x_{14}=x_{41}$ is the sum of five Laurent monomials, one for each Catalan path from node 1 to node 4. The last term $\frac{a_{13 \mid 2} a_{23} a_{24 \mid 3}}{p_{2} p_{23} p_{3}}$ equals $W_{\mathscr{C}}(C)$ for the path $C$ shown in Figure 1

The proof of Theorem 1.1 is given in Section 4 . We start in Section 2 by reviewing a theorem of Kenyon and Pemantle [3] which expresses the entries of an arbitrary square matrix in terms of almost-principal and principal minors, as a sum of Laurent monomials that are in bijection with domino tilings of a half Aztec diamond. In Section 3, we give a bijection between these domino tilings and Schröder paths, and restate their theorem using Schröder paths. We then prove our theorem by constructing a projection from Schröder paths to Catalan paths and applying the relation (6) among minors of symmetric matrices.

In Section 5 we connect Theorem 1.1 to an application in statistics, developed in work of Joe, Kurowicka and Lewandowski [2, 5]. Namely, we focus on symmetric matrices that are positive definite and have all diagonal entries equal to 1 . These are the correlation matrices, and they form a convex set that is known in optimization as the elliptope [1, 4]. Our formula yields an explicit bijection between the elliptope and the open cube $(-1,1)\left(\begin{array}{c}n \\ 2\end{array}\right)$.

\section{Square matrices and tilings of the half Aztec diamond}

In this section we review the Kenyon-Pemantle formula in [3, Theorem 4.4]. The half Aztec diamond $H D_{n}$ of order $n$ is the union of the unit squares whose vertices are in the set

$$
\left\{(a, b) \in \mathbb{Z}^{2}:|a| \leq n, 0 \leq b \leq n,|a|+|b| \leq n+1\right\} .
$$

We label the boxes in the bottom row of $H D_{n}$ by the numbers 1 through $2 n$, from left to right. We label certain lattice points of $H D_{n}$ by minors as follows. Fix $b \in[n]$. The connected principal minors $p_{I}$ such that $|I|=b$ are assigned to the lattice points $(a, b)$ with $a+b$ even. The connected almost-principal minors $a_{i j \mid I}$ with $i>j$ and $|I|=b-1$ are assigned to the lattice points $(a, b)$ with $a+b$ odd. In both cases, the assignment is from left to right using the lexicographic order on $I$. The case $n=4$ is shown in Figure 2 .

Fix integers $a$ and $b$ such that $a$ is even, $b$ is odd, and $1<a<b<2 n$. We define the colored half Aztec diamond $H D_{n}(a, b)$ by coloring the boxes of $H D_{n}$ black, grey, or white. First color boxes $a$ and $b$ in the bottom row black. Let $L_{a}$ be the diagonal line of slope 1 through box $a-1$, and let $L_{b}$ be the line of slope -1 through box $b+1$. If a box (or any part of it) lies to the left of $L_{a}$ or to the right of $L_{b}$, then color it grey. All other boxes are white. A domino tiling (or simply a tiling) of $H D_{n}(a, b)$ is a tiling of the white boxes by $1 \times 2$ and $2 \times 1$ rectangles. Let $\mathscr{A}_{n}(a, b)$ denote the set of tilings of $H D_{n}(a, b)$. Figure 3 shows the set $\mathscr{A}_{4}(2,7)$, i.e. the six tilings of $H D_{4}(2,7)$, with lines $L_{2}$ and $L_{7}$ superimposed on the tilings.

Each tiling $T$ of the colored half Aztec diamond $H D_{n}(a, b)$ gets a Laurent monomial weight, which we now define. We regard $T$ as a simple graph whose nodes are the lattice points of $H D_{n}$, and whose edges are induced by the edges of the rectangles in the tiling together with the edges of the unit squares outside 


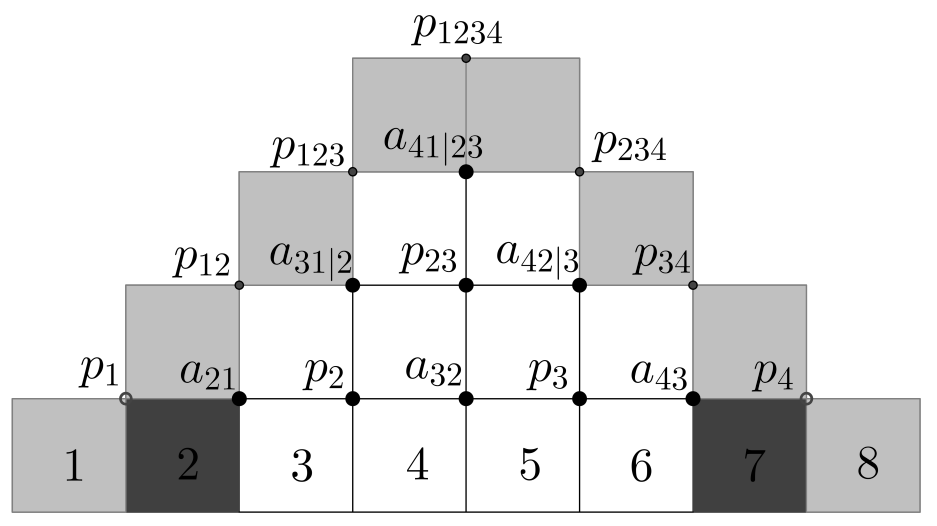

Fig. 2: The half Aztec diamond $H D_{4}$. The white boxes are to be tiled.

the tiling. An interior lattice point of $H D_{n}(a, b)$ is a lattice point which lies strictly to the right of $L_{a}$ and strictly to the left of $L_{b}$. The interior lattice points that will concern us are shown in bold in Figures 2 and 3 Each of these is labeled by a variable $v_{\ell}$ which is a connected principal or almost-principal minor. The weight $W_{\mathscr{A}}(T)$ of a tiling $T \in \mathscr{A}_{n}(a, b)$ is defined to be the Laurent monomial

$$
W_{\mathscr{A}}(T):=\prod_{\ell} v_{\ell}^{d(\ell)-3},
$$

where $\ell$ ranges over the interior lattice points of $H D_{n}(a, b)$ and $d(\ell)$ is the degree of $\ell$ in $T$.

Theorem 2.1 (Kenyon-Pemantle [3]) The entries of an $n \times n$ matrix $X=\left(x_{i j}\right)$ satisfy

$$
x_{i j}=\sum_{T \in \mathscr{A}_{n}(2 j, 2 i-1)} W_{\mathscr{A}}(T) \quad \text { for } i>j .
$$

Theorem 4.4 in [3] also gives a similar formula for $x_{i j}$ with $i<j$, but we omit that formula, as it is not needed here.

Example 2.2 Figure 3 shows the six tilings of $H D_{4}(2,7)$ with their weights. By Theorem 2.1 the upper right matrix entry for $n=4$ is the sum of these six Laurent monomials:

$$
x_{41}=\frac{a_{21} a_{32} a_{43}}{p_{2} p_{3}}+\frac{a_{31 \mid 2} a_{43}}{p_{2} p_{3}}+\frac{a_{21} a_{42 \mid 3}}{p_{2} p_{3}}+\frac{a_{31 \mid 2} a_{42 \mid 3}}{p_{2} p_{3} a_{32}}+\frac{a_{31 \mid 2} a_{42 \mid 3}}{p_{23} a_{32}}+\frac{a_{41 \mid 23}}{p_{23}} .
$$

The full $4 \times 4$ matrix is shown on page 8 of [3], albeit with different notation.

\section{Square matrices and Schröder paths}

In this section we continue our discussion of arbitrary square matrices. A Schröder path $S$ is a path in the $x y$-plane which starts at $(0,0)$, always stays at or above the $x$-axis, and consists of steps which are 

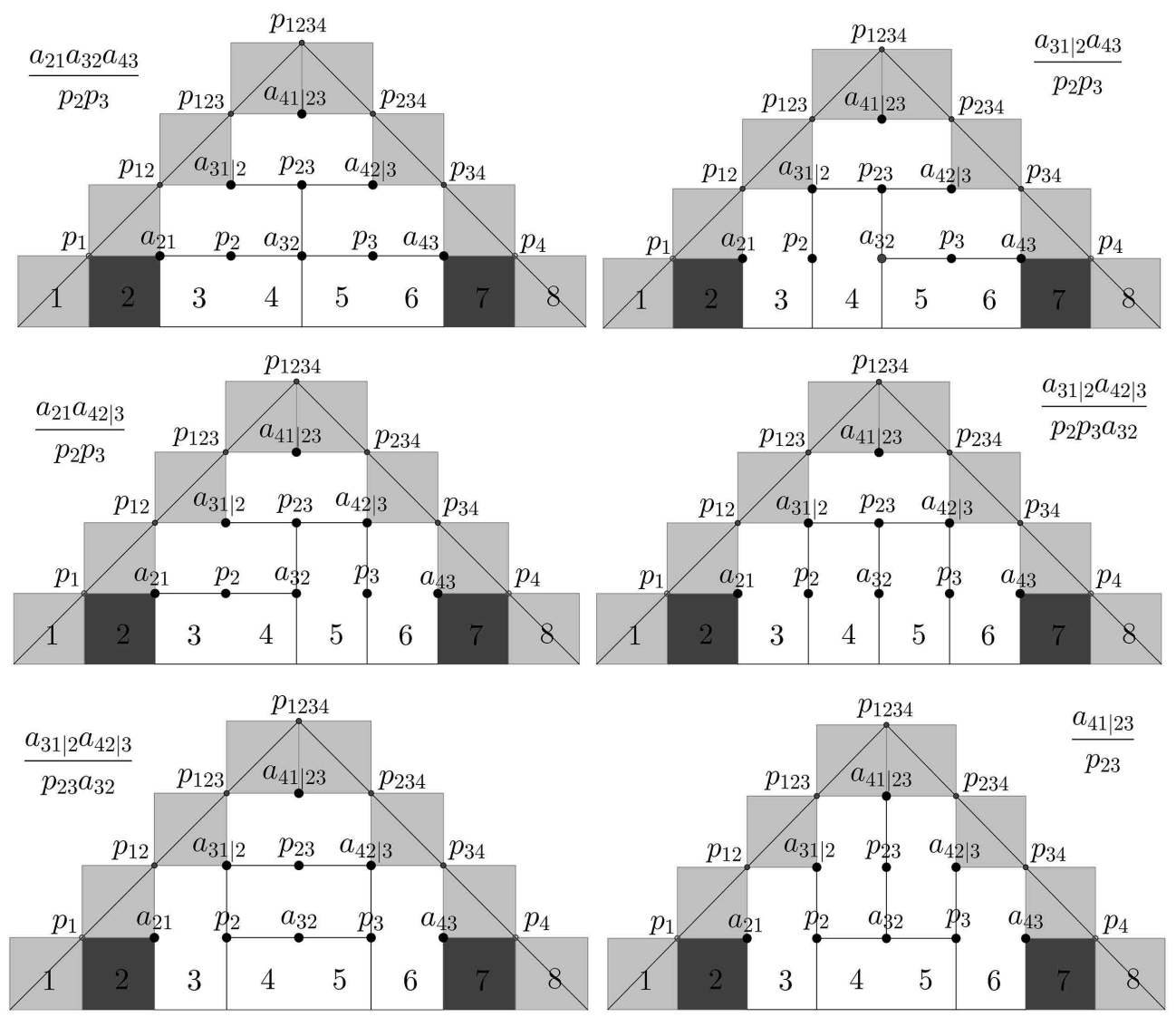

Fig. 3: The six tilings of the colored half Aztec diamond $H D_{4}(2,7)$.

either northeast $(1,1)$, southeast $(1,-1)$, or horizontal $(2,0)$. A Schröder path has order $n$ if it ends at $(2 n-4,0)$. Let $G_{n}^{\prime}$ denote the planar graph whose nodes are the lattice points $(x, y)$ with $0 \leq y \leq x$ and $x+y \leq 2 n-4$ even, with edges given by northeast, southeast and horizontal steps. The set $\mathscr{S}_{n}$ of Schröder paths of order $n$ is identified with the left-to-right paths in $G_{n}^{\prime}$ from $(0,0)$ to $(2 n-4,0)$. The cardinality of $\mathscr{S}_{n}$ is the Schröder number, which is given by the generating function [7]

$$
\sum_{n=2}^{\infty}\left|\mathscr{S}_{n}\right| z^{n-2}=\frac{1-z-\sqrt{1-6 z+z^{2}}}{2 z}=1+2 z+6 z^{2}+22 z^{3}+90 z^{4}+394 z^{5}+1806 z^{6}+\cdots
$$

The graph $G_{n}^{\prime}$ is labeled by connected minors. We assign $a_{i j \mid I}$ to the node $(i+j-3, i-j-1)$ for $i>j$, and we assign $p_{I}$ to the triangle below that node. We refer to $(2 i-2,0)$ as node $i$. Figure 4 shows the case $n=4$. The six Schröder paths in $\mathscr{S}_{4}$ are shown in Figure 6

We now define the weight $W_{\mathscr{S}}(S)$ of a Schröder path $S$ on $G_{n}^{\prime}$. We regard $S$ as a graph with vertices 


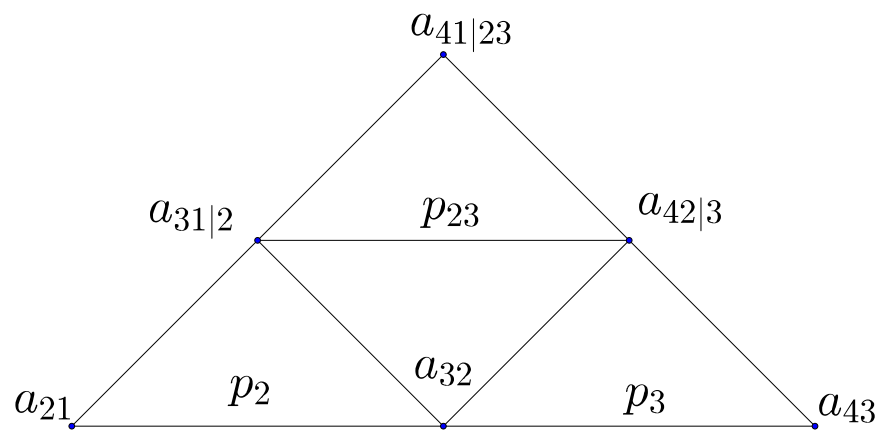

Fig. 4: The graph $G_{4}^{\prime}$ encodes the Schröder paths of order 4 .

$V(S)$ and edges $E(S)$. Given a Schröder path $S$ on $G_{n}^{\prime}$, we define the sets

$$
\begin{aligned}
& \alpha(S)=\{v \in V(S): v \text { is a weak local maximum of } S\}, \\
& \beta(S)=\{e \in E(S): e \text { is immediately below a weak local minimum of } S\}, \\
& \gamma(S)=\{e \in E(S): e \text { is a horizontal edge of } S\}, \\
& \delta(S)=\{v \in V(S): v \text { is immediately below a horizontal edge of } S\}, \\
& \epsilon(S)=\{e \in E(S): e \text { is immediately below a strict local maximum of } S\}, \\
& \zeta(S)=\quad\{v \in V(S): v \text { is a strict local minimum (but not an endpoint) of } S\} .
\end{aligned}
$$

Each of these is regarded as a monomial by taking the product of all labels. Then we define

$$
W_{\mathscr{S}}(S)=\frac{\alpha(S) \beta(S)}{\gamma(S) \delta(S) \epsilon(S) \zeta(S)} .
$$

Figure 6 shows the six Schröder paths for $n=4$, together with their weights. The sum of these weights is the Laurent polynomial in (3), which evaluates to the matrix entry $x_{41}$.

The main result of this section is a reformulation of Theorem 2.1 in terms of Schröder paths. We write $\mathscr{S}_{n}(a, b)$ for the set of all Schröder paths from node $a$ to node $b$ in $G_{n}^{\prime}$.

Theorem 3.1 The entries of an $n \times n$ matrix $X=\left(x_{i j}\right)$ satisfy

$$
x_{i j}=\sum_{S \in \mathscr{S}_{n}(j, i-1)} W_{\mathscr{S}}(S) \quad \text { for } i>j \text {. }
$$

We shall present a weight-preserving bijection $\Phi: \mathscr{A}_{n}(2 j, 2 i-1) \rightarrow \mathscr{S}_{n}(j, i-1)$ between tilings and Schröder paths. Note that we can superimpose the graph $G_{n}^{\prime}$ on the graph $H D_{n}$ so that the labels (connected minors) match up. When we do this, the vertex $j$ (respectively, $i-1$ ) of $G_{n}^{\prime}$ gets identified with the top right corner of the square $2 j$ (respectively, the top left corner of the square $2 i-1$ ) in $H D_{n}$. We draw a Schröder path $\Phi(T)$ on top of a tiling $T$, as in Figure 5 . We may then think of the path as an element of $\mathscr{S}_{n}(j, i-1)$. 

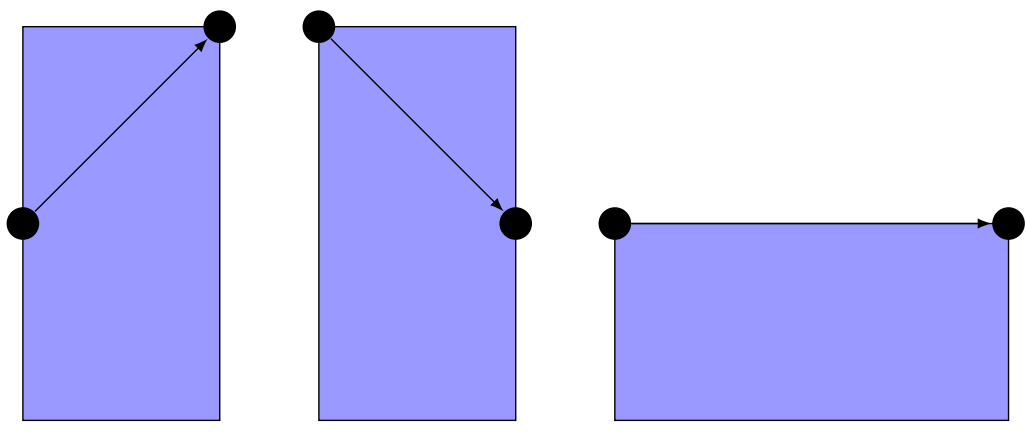

Fig. 5: How to construct a Schröder path from a tiling.

More formally, given $T \in \mathscr{A}_{n}(2 j, 2 i-1)$, the path $\Phi(T) \in \mathscr{S}_{n}(j, i-1)$ is defined as follows. Its starting point is the top right corner of square $2 j$ in $H D_{n}(2 j, 2 i-1)$. We inductively add steps to $\Phi(T)$ depending on the local behavior of the tiling, as shown in Figure 5 . Let $x$ denote the endpoint of the path that we have built so far. Then we proceed as follows:

- If there is a vertical tile to the east of $x$, then we add a northeast step to our path.

- If there is a vertical tile to the southeast of $x$, such that $x$ is at its northwest corner, then we add a southeast step to our path.

- If there is a horizontal tile to the southeast of $x$, then add an east step to our path.

- If $x$ is already at the top left corner of square $2 i-1$, then we stop.

The map $\Phi$ maps the six tilings in Figure 3 to the six Schröder paths in Figure 6

Lemma 3.2 The map $\Phi: \mathscr{A}_{n}(2 j, 2 i-1) \rightarrow \mathscr{S}_{n}(j, i-1)$ is well-defined and is a bijection. Moreover, if $T$ is a tiling in $\mathscr{A}_{n}(2 j, 2 i-1)$, where $i>j$, then $W_{\mathscr{S}}(\Phi(T))=W_{\mathscr{A}}(T)$.

Thus this bijection is weight-preserving.

\section{Back to symmetric matrices}

The strategy for proving Theorem 1.1 is to combine Theorem 3.1 with a projection from Schröder paths to Catalan paths. Let $S$ be any Schröder path in $G_{n}^{\prime}$. The associated Catalan path $\pi(S)$ in $G_{n}$ is defined by

- replacing each horizontal step in $S$ with a strict local minimum, i.e. a southeast step followed by a northeast step;

- adding a northeast step at the beginning of $S$ and a southeast step at the end of $S$.

If $S$ starts at $i$ and ends at $j-1$ in $G_{n}^{\prime}$ then $\pi(S)$ starts at $i$ and ends at $j$ in $G_{n}$. Figure 7 shows how four of the six Schröder paths in $\mathscr{S}_{4}(1,3)$ map to four of the five Catalan paths in $\mathscr{C}_{4}(1,4)$. The two other Schröder paths in Figure 6 map to the Catalan path in Figure 1 

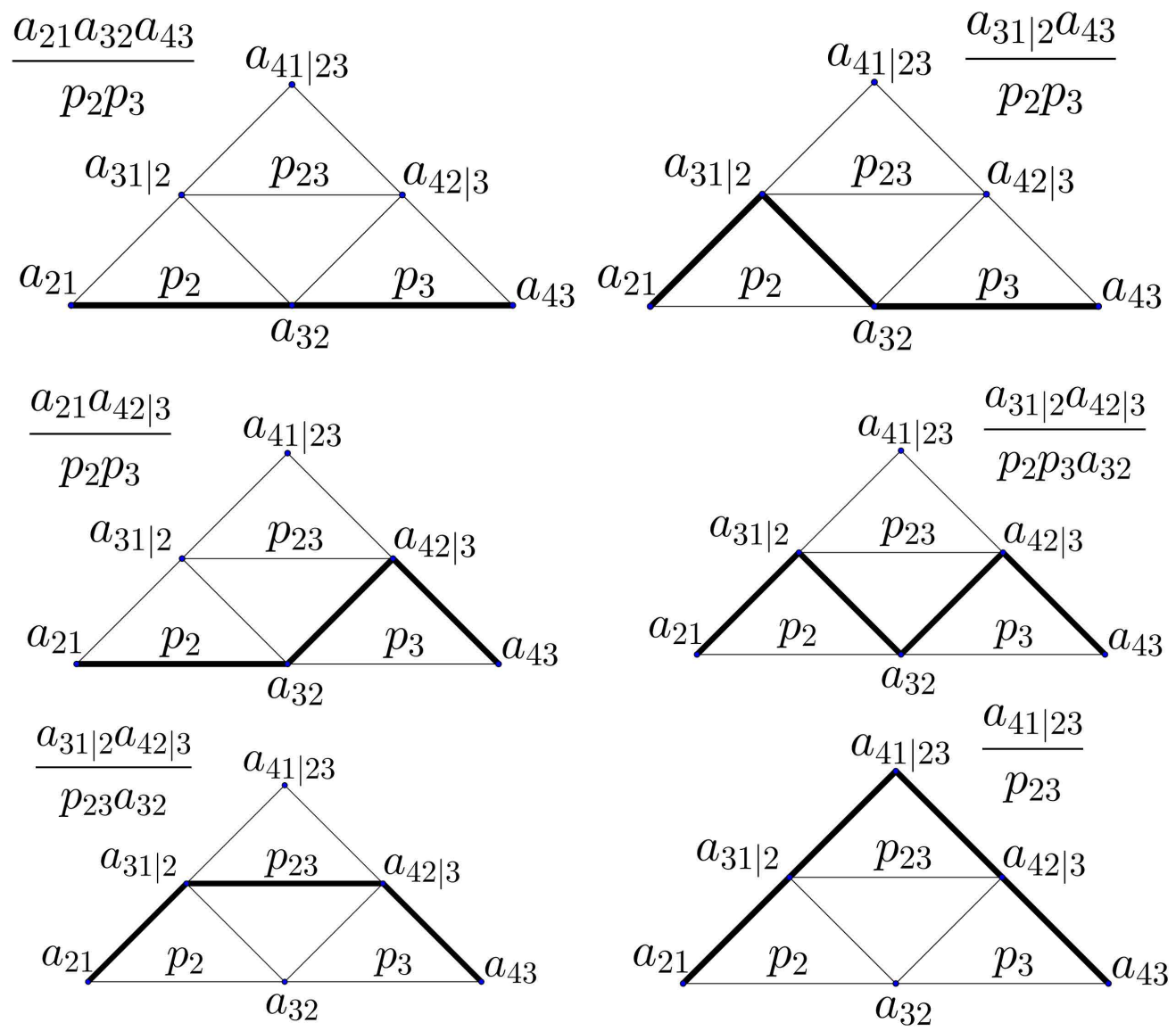

Fig. 6: The six Schröder paths in $\mathscr{S}_{4}$ together with their weights.

Proposition 4.1 Assume that the labels of the graphs come from a symmetric matrix. The weight of a Catalan path is the sum of the weights of the Schröder paths in its preimage under the projection $\pi$, i.e.

$$
\sum_{S \in \pi^{-1}(C)} W_{\mathscr{S}}(S)=W_{\mathscr{C}}(C) .
$$

An important ingredient in the proof is the following identity that expresses connected almost-principal minors of a symmetric $n \times n$ matrix in terms of connected principal minors:

$$
a_{i j \mid I}^{2}-p_{I} p_{I \cup\{i, j\}}-p_{I \cup\{i\}} p_{I \cup\{j\}}=0, \quad 2 \leq i<j \leq n-1, I=\{i+1, \ldots, j-1\} .
$$

Example 4.2 Let $S^{\prime}$ and $S$ be the fourth and fifth Schröder paths in Figure 6, with labels given by a symmetric $4 \times 4$ matrix. Using the identity $a_{23}=p_{23}+p_{2} p_{3}$, as in 6 , we find

$$
W_{\mathscr{S}}(S)+W_{\mathscr{S}}\left(S^{\prime}\right)=\frac{a_{13 \mid 2} a_{24 \mid 3}}{p_{2} p_{3} a_{23}}+\frac{a_{13 \mid 2} a_{24 \mid 3}}{p_{23} a_{23}}=\frac{a_{13 \mid 2} a_{24 \mid 3} a_{23}}{p_{2} p_{23} p_{3}} .
$$


This explains how the six terms in (3) become the five terms of $x_{14}$ shown in (2). Namely, the weight of the Catalan path in Figure 1 is the sum of the fourth and fifth terms in (3).

Remark 4.3 The expression in Theorem 1.1 is not the only way to express the entries of a symmetric matrix in terms of the $\left(\begin{array}{c}n \\ 2\end{array}\right)+\left(\begin{array}{c}n-2 \\ 2\end{array}\right)+n$ connected almost-principal and principal minors. The prime ideal of polynomial relations among these minors is generated by the $\left(\begin{array}{c}n-2 \\ 2\end{array}\right)$ quadrics in (6). To show this, we argue as follows. First, in Theorem 1.1 we have expressed the $\left(\begin{array}{c}n+1 \\ 2\end{array}\right)$ algebraically independent matrix entries $x_{i j}$ in terms of these minors. This ensures that the algebra generated by these minors has Krull dimension $\left(\begin{array}{c}n+1 \\ 2\end{array}\right)$. Hence their relation ideal has codimension $\left(\begin{array}{c}n-2 \\ 2\end{array}\right)=\left(\begin{array}{c}n \\ 2\end{array}\right)+\left(\begin{array}{c}n-2 \\ 2\end{array}\right)+n-\left(\begin{array}{c}n+1 \\ 2\end{array}\right)$. The $\left(\begin{array}{c}n-2 \\ 2\end{array}\right)$ relations 6 lie in that ideal and they generate a complete intersection. Our final claim is that this complete intersection is a prime ideal. We deduce this from the fact that none of the $a_{i j \mid I}$ has a square root in the subalgebra generated by the principal minors. For a concrete example consider $n=4$. Here, our ideal of relations is the principal ideal $\left\langle a_{23}^{2}-p_{2} p_{3}-p_{23}\right\rangle$.

\section{Parametrizing Correlation Matrices}

We now specialize to real symmetric $n \times n$ matrices that are positive definite and have all diagonal entries equal to 1 . Such matrices are known as correlation matrices. They play an important role in statistics, notably in the study of multivariate normal distributions. The set $\mathcal{E}_{n}$ of all $n \times n$ correlation matrices is an open convex set of dimension $\left(\begin{array}{l}n \\ 2\end{array}\right)$. Its closure is a convex body, known in optimization theory [1, 4] under the name elliptope.

In certain statistical applications it is desirable to generate random correlation matrices. Specifically, one wishes to sample from the uniform distribution on the elliptope $\mathcal{E}_{n}$. A solution to this problem was given by Joe [2] and further refined by Lewandowski et al. [5]. The underlying geometric idea is to construct a parametrization from the standard cube:

$$
\Psi:(-1,1)^{\left(\begin{array}{l}
n \\
2
\end{array}\right)} \rightarrow \mathcal{E}_{n}
$$

The papers [2, 5] describe such maps $\Psi$ that are algebraic and bijective, so they identify the open cube with the open elliptope. However, the construction is recursive. In what follows we revisit the formula in [2] and we make it completely explicit. Remarkably, it is precisely the restriction of our Laurent polynomial parametrization in Theorem 1.1 to the region where all connected principal minors $p_{I}$ are positive and $p_{1}=\cdots=p_{n}=1$.

Let $X=\left(x_{i j}\right)$ be a real symmetric $n \times n$ matrix. We assume that $X$ is positive definite, i.e. all principal minors $p_{I}$ are strictly positive. In statistics, such an $X$ serves as the covariance matrix of a normal distribution on $\mathbb{R}^{n}$, whose partial correlations are given by

$$
\rho_{i j \mid I}=\frac{(-1)^{\lceil|I| / 2\rceil} \cdot a_{i j \mid I}}{\sqrt{p_{i I} \cdot p_{j I}}} \quad \text { where } i, j \notin I \text { and } i<j .
$$

For $I=\emptyset$, we obtain the $\left(\begin{array}{l}n \\ 2\end{array}\right)$ entries of the correlation matrix $Y=\left(y_{i j}\right)$, namely

$$
y_{i j}=\rho_{i j}=\frac{a_{i j}}{\sqrt{p_{i} p_{j}}}=\frac{x_{i j}}{\sqrt{x_{i i} x_{j j}}} \quad \text { for } 1 \leq i<j \leq n \text {. }
$$

The partial correlation $\rho_{i j \mid I}$ in (7) is called connected if $I=\{i+1, i+2, \ldots, j-2, j-1\}$. 
Theorem 5.1 The $\left(\begin{array}{l}n \\ 2\end{array}\right)$ entries $y_{i j}$ of a correlation matrix can be written uniquely in terms of the $\left(\begin{array}{l}n \\ 2\end{array}\right)$ connected partial correlations $\rho_{i j \mid I}$. Explicit formulas are derived from those in Theorem 1.1 by first replacing each occurrence of a parameter $a_{i j \mid I}$ by $(-1)^{\lceil|I| / 2\rceil} \rho_{i j \mid I} \sqrt{p_{i I} p_{j I}}$ and thereafter replacing each occurrence of a parameter $p_{r, r+1, \ldots, s}$ by the product of the $\left(\begin{array}{c}s-r+1 \\ 2\end{array}\right)$ expressions $(-1)^{\lfloor|I| / 2\rfloor}(1-$ $\left.\rho_{i j \mid I}^{2}\right)$ where $r \leq i<j \leq s$ and $I=\{i+1, i+2, \ldots, j-1\}$. The resulting map $\Psi:\left(\rho_{i j \mid I}\right) \mapsto\left(y_{i j}\right)$ is a bjection between $(-1,1)^{\left(\begin{array}{l}n \\ 2\end{array}\right)}$ and $\mathcal{E}_{n}$.

We now illustrate our parametrization of correlation matrices in the two smallest cases.

Example 5.2 $(n=3)$ We consider the open 3-dimensional cube defined by the inequalities

$$
-1<\rho_{12}, \rho_{23}, \rho_{13 \mid 2}<1 .
$$

Our bijection $\Psi$ identifies each point in this cube with a $3 \times 3$ correlation matrix:

$$
\left[\begin{array}{ccc}
1 & y_{12} & y_{13} \\
y_{12} & 1 & y_{23} \\
y_{13} & y_{23} & 1
\end{array}\right]=\left[\begin{array}{ccc}
1 & \rho_{12} & \rho_{12} \rho_{23}-\rho_{13 \mid 2}\left(1-\rho_{12}^{2}\right)^{\frac{1}{2}}\left(1-\rho_{23}^{2}\right)^{\frac{1}{2}} \\
\rho_{12} & 1 & \rho_{23} \\
\rho_{12} \rho_{23}-\rho_{13 \mid 2}\left(1-\rho_{12}^{2}\right)^{\frac{1}{2}}\left(1-\rho_{23}^{2}\right)^{\frac{1}{2}} & \rho_{23} & 1
\end{array}\right] .
$$

One checks that this matrix is positive definite, and, as in [2, Theorem 1], its determinant

$$
\operatorname{det}(Y)=\left(1-\rho_{12}^{2}\right)\left(1-\rho_{23}^{2}\right)\left(1-\rho_{13 \mid 2}^{2}\right)
$$

defines the facets of the cube. It is instructive to draw how the boundary of the cube maps onto the boundary of the elliptope $\mathcal{E}_{3}$. The latter is depicted in [1, Figure 5.8, page 232].

The combinatorics of our planar graph $G_{n}$ and its Catalan paths can be seen in a different guise in [2. 5]. These correspond to the structures called $D$-vines in these papers. Figure 8 shows the standard D-vine for $n=4$. Its edges are naturally labeled with the six coordinates of the cube, namely $\rho_{12}, \rho_{23}, \rho_{34}, \rho_{13 \mid 2}, \rho_{24 \mid 3}, \rho_{14 \mid 23}$. These correspond to the six almost-principal minors $a_{i j \mid I}$ in the labeled graph $G_{4}$ in Figure 1 .

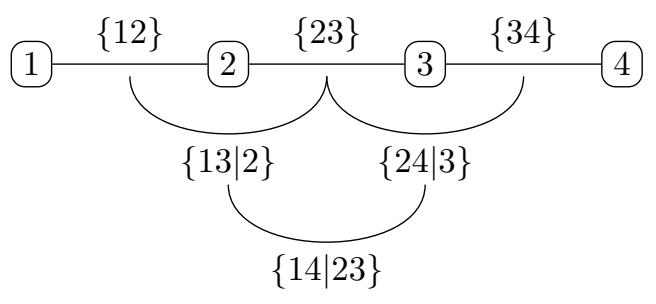

Fig. 8: The standard D-vine for four random variables.

\section{Acknowledgements}

We thank Richard Kenyon, Robin Pemantle and Caroline Uhler for helpful conversations. This project was supported by the National Science Foundation (DMS-1419018, DGE-1106400, DMS-1049513). 


\section{References}

[1] Grigoriy Blekherman, Pablo Parrilo and Rekha Thomas: Semidefinite Optimization and Convex Algebraic Geometry, MOS-SIAM Ser. Optim., 13, SIAM, Philadelphia, PA, 2013.

[2] Harry Joe: Generating random correlation matrices based on partial correlations, J. Multivariate Anal., 97(10):2177-2189, 2006.

[3] Richard Kenyon and Robin Pemantle: Principal minors and rhombus tilings, J. Phys. A: Math. Theor., 47:474010, 2014.

[4] Monique Laurent and Svatopluk Poljak: On the facial structure of the set of correlation matrices, SIAM J. Matrix Anal. Appl. 17: 530-547, 1996.

[5] Daniel Lewandowski, Dorota Kurowicka, and Harry Joe: Generating random correlation matrices based on vines and extended onion method, J. Multivariate Anal., 100(9):1989-2001, 2009.

[6] Nicolau Saldanha and Carlos Tomei: An overview of domino and lozenge tilings, Resenhas IME-USP, 2(2):239-252, 1995.

[7] Richard P. Stanley: Enumerative Combinatorics, Vol. 2, volume 62 of Cambridge Studies in Advanced Mathematics, Cambridge University Press, 1999. 

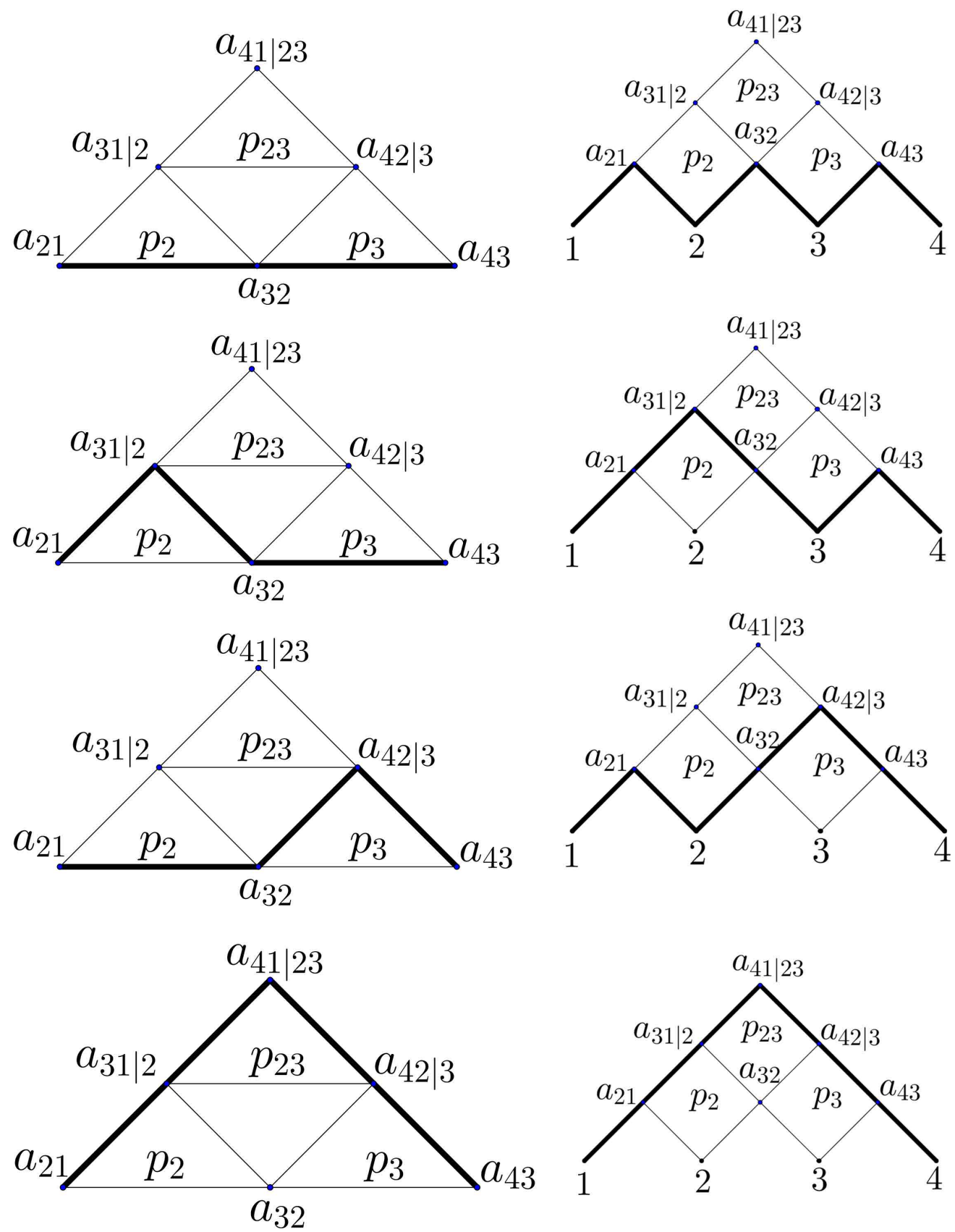

Fig. 7: The Schröder paths (left) are projected to the Catalan paths (right). 